\title{
Gravitational field of vacuumless defects
}

\author{
Inyong Cho* and Alexander Vilenkin ${ }^{\dagger}$ \\ Institute of Cosmology, Department of Physics and Astronomy, \\ Tufts University, Medford, Massachusetts 02155, USA
}

(May 31, 2022)

\begin{abstract}
It has been recently shown that topological defects can arise in symmetry breaking models where the scalar field potential $V(\phi)$ has no minima and is a monotonically decreasing function of $|\phi|$. Here we study the gravitational fields produced by such vacuumless defects in the cases of both global and gauge symmetry breaking. We find that a global monopole has a strongly repulsive gravitational field, and its spacetime has an event horizon similar to that in de Sitter space. A gauge monopole spacetime is essentially that of a magnetically charged black hole. The gravitational field of a global string is repulsive and that of a gauge string is attractive at small distances and repulsive at large distances. Both gauge and global string spacetimes have singularities at a finite distance from the string core.
\end{abstract}

PACS number(s): $98.80 . \mathrm{Cq}$

*Electronic address: cho@cosmos2.phy.tufts.edu

†Electronic address: vilenkin@cosmos2.phy.tufts.edu 


\section{INTRODUCTION}

Topological defects are high-energy relics which could be formed at symmetry-breaking phase transitions in the early universe [1.2]. A prototypical symmetry breaking model is described by the Lagrangian

$$
\mathcal{L}=\frac{1}{2} \partial_{\mu} \phi_{a} \partial^{\mu} \phi_{a}-V(f)
$$

where $\phi_{a}$ is a set of scalar fields, $a=1, \ldots, N, f=\left(\phi_{a} \phi_{a}\right)^{1 / 2}$, and the potential $V(f)$ has a minimum at a non-zero value of $f$. The model has $\mathrm{O}(\mathrm{N})$ symmetry and admits domain wall, string, and monopole solutions for $N=1,2$, and 3 , respectively.

One can consider a different class of models in which $V(f)$ has a local maximum at $f=0$ but no minima; instead, it monotonically decreases to zero at $f \rightarrow \infty$. For example,

$$
V(f)=\lambda M^{4+n}\left(M^{n}+f^{n}\right)^{-1} .
$$

In the particle physics context, potentials without stable vacua can arise due to nonperturbative effects in supersymmetric theories [3]. Models of this sort have attracted considerable interest in cosmology, where the "rolling" field $\phi$ may significantly contribute to the present energy density of the universe [⿶凵⿴囗十 .

In a recent paper [5] we have studied the properties of topological defects in models of the type (1.1), (1.2), for both gauge and global symmetries. Since the potential $V(f)$ does not have a stable vacuum and decreases monotonically with $f$, the scalar field magnitude $f(r)$ grows unboundedly with the distance $r$ from the defect core. We have introduced the term "vacuumless" for defects of this sort. The defect solutions of Ref. [5] have been found in flat spacetime. The purpose of present paper is to study the gravitational field of vacuumless defects.

Let us first summarize the relevant conclusions of Ref. [5].

(i) For a vacuumless domain wall centered at $x=0$, the asymptotic behavior of the scalar

field and of the energy density is $f(x) \propto|x|^{2 /(n+2)}$ and $T_{0}^{0}(x) \propto|x|^{-2 n /(n+2)}$ at $|x| \rightarrow \infty$. For 
$n>2$, the energy distribution is integrable, with most of the energy concentrated near the wall mid-section $(x=0)$. We expect, therefore, that the gravitational field of vacuumless walls will not be much different from that of ordinary domain walls.

(ii) For global vacuumless strings and monopoles, the flat-space solution is

$$
f(r)=a M(r / \delta)^{\frac{2}{n+2}},
$$

where $\delta=\lambda^{-1 / 2} M^{-1}$ is the size of the defect core, $r$ is the distance from the monopole center for monopoles and the distance from the string axis for strings, and $a \sim 1$ is a numerical coefficient, $a=(n+2)^{2 /(n+2)}(n+4)^{-1 /(n+2)}$ for strings and $a=(n+2)^{2 /(n+2)}[2(n+3)]^{-1 /(n+2)}$ for monopoles. The solution (1.3) applies for $\delta \ll r \ll R$, where $R$ is the cutoff radius determined by the distance to the nearest string or monopole. Vacuumless strings and monopoles are very diffuse objects, with most of their energy distributed at large distances from the core $(r \sim R)$, and with potential and gradient energy contributing comparable amounts. They are much more diffuse than ordinary strings and monopoles, and we expect their spacetimes to be substantially different from the ordinary case.

(iii) Gauge vacuumless strings have magnetic flux localized within a thin tube inside the core, while outside the core the scalar field is given by

$$
f(r)=a \ln (r / \delta)+b .
$$

An unusual feature of this solution is that the coefficients $a$ and $b$ are sensitive to the cutoff distance $R$,

$$
a \sim M(R / \delta)^{\frac{2}{n+2}}[\ln (R / \delta)]^{-\frac{n+1}{n+2}}, \quad b \sim a \ln (R / \delta) .
$$

The energy distribution at small distances is dominated by the gradient energy with $T_{0}^{0} \propto$ $r^{-2}$, while the potential energy begins to dominate at $r \gtrsim R / \ln ^{1 / 2}(R / \delta)$.

(iv) Finally, a flat-space vacuumless gauge monopole is described by the Prasad-Sommerfield solution. For $r \ll R$, the scalar field is given by 


$$
f(r)=a \operatorname{coth}(e a r)-1 / e r
$$

where

$$
a \sim M\left(\lambda e M^{3} R^{3}\right)^{1 /(n+1)}
$$

and $e$ is the gauge coupling. The size of the monopole core is $r_{0} \sim 1 / e a$. Much of the monopole energy is concentrated near the core; the total energy in that region is

$$
\mathcal{M}=\frac{4 \pi}{e} a
$$

In addition, there is a nearly constant energy density $T_{0}^{0} \approx V(a)$ outside the core. Its contribution to the total energy is comparable to (1.8), $V(r) R^{3} \sim \mathcal{M}$.

In the next section, the spacetimes of vacuumless defects will be studied using the linearized gravity approximation. Numerical solutions of the full Einstein's, scalar, and gauge field equations will be discussed in Sec. III.

\section{LINEARIZED GRAVITY}

We shall first consider the Newtonian approximation which applies for weak gravitational fields and non-relativistic motion. The Newtonian potential $\Phi$ can be found for the equation

$$
\nabla^{2} \Phi=4 \pi G\left(T_{0}^{0}-T_{i}^{i}\right)
$$

For a global defect described by the Lagrangian (1.1),

$$
T_{\mu}^{\nu}=\partial_{\mu} \phi_{a} \partial^{\nu} \phi_{a}-\delta_{\mu}^{\nu} \mathcal{L}
$$

and, for a static field $\phi_{a}(\mathrm{x})$,

$$
T_{0}^{0}-T_{i}^{i}=-2 V(f)
$$

For global vacuumless strings and monopoles, $f(r)$ is given by Eq. (1.3) and

$$
T_{0}^{0}-T_{i}^{i}=-\frac{2 \lambda M^{4}}{a^{n}}\left(\frac{\delta}{r}\right)^{\frac{2 n}{n+2}} .
$$


The solution of Eq. (2.1) is then

$$
\Phi(r)=-K G M^{2}(r / \delta)^{4 /(n+2)}
$$

where $K \sim 1$ is a numerical coefficient, $K=2 \pi(n+2)^{2} / a^{n}(n+6)$ for monopoles and $K=\pi(n+2)^{2} / 2 a^{n}$ for strings. One could also include a solution of the homogeneous equation, $\Phi_{0}(r)$, representing the gravitational field produced by the defect core $\left(\Phi_{0} \propto r^{-1}\right.$ for monopoloes and $\Phi_{0} \propto \ln (r / \delta)$ for strings), but it becomes subdominant at sufficiently large $r$.

The potential (2.5) describes a strong repulsive gravitational field. This is not surprising, considering the sign of the source in Eq. (2.3). Note that for $n=2$ the repulsive force is independent of the distance from the defect core. The linearized approximation applies as long as $\Phi(r) \ll 1$. From (1.3) and (2.5) we see that this is equivalent to $f(r) \ll m_{p}$, where $m_{p}=G^{-1 / 2}$ is the Planck mass.

For a gauge string, the gauge field is negligible outside the core, the energy momentum tensor (2.2) is modified by replacing ordinary derivatives of $\phi_{a}$ by gauge-covariant derivatives, and Eq. (2.3) is still valid. However, in this case the potential energy is negligible compared to the gradient energy, provided that $r$ is not too large, $r \ll R / \ln ^{1 / 2}(R / \delta)$. The gravitational potential in this range of $r$ should then be given by a solution of the homogeneous equation, $\Phi(r) \propto \ln (r / \delta)$.

We now turn to linearized Einstein's gravity. The gravitational field of a vacuumless global monopole is described by a static, spherically-symmetric metric which can be represented as

$$
d s^{2}=-B(r) d t^{2}+A(r) d r^{2}+r^{2} d \Omega^{2}
$$

where $d \Omega^{2}$ is the metric on a unit 2-sphere. Einstein's equations for this metric are given in the Appendix. Expressing $A$ and $B$ as

$$
A(r)=1+\alpha(r), \quad B(r)=1+\beta(r),
$$


linearizing in $\alpha$ and $\beta$, and using the flat space expression (1.3) for $f(r)$, the equations are reduced to

$$
\begin{gathered}
\beta^{\prime \prime}+\frac{2}{r} \beta^{\prime}=-\frac{16 \pi \lambda}{a^{n}} G M^{4}\left(\frac{\delta}{r}\right)^{\frac{2 n}{n+2}}, \\
\frac{\alpha^{\prime}}{r}+\frac{\beta^{\prime}}{r}=-8 \pi \lambda\left(\frac{2}{n+2}\right)^{2} a^{2} G M^{4}\left(\frac{\delta}{r}\right)^{\frac{2 n}{n+2}} .
\end{gathered}
$$

The solution is easily found and can be expressed in the form

$$
d s^{2}=-(1+2 \Phi) d t^{2}+(1+k \Phi) d r^{2}+r^{2} d \Omega^{2}
$$

where $\Phi(r)$ is the Newtonian potential (2.5) and $k \sim 1$ is a numerical coefficient. The coefficients $k$ and $K$ are functions of $n$ given by rather complicated expressions which we shall not reproduce here. In the special case of $n=2$ which we shall use in our numerical examples, $a=2 / 10^{1 / 4}=1.13, K=3 \pi$, and $k=17 / 16$.

For a global vacuumless string, we assume that the spacetime is static, cylindricallysymmetric, and also has a symmetry with respect to Lorentz boosts along the string axis. With a suitable choice of coordinates, the metric can be written as

$$
d s^{2}=B(r)\left(-d t^{2}+d z^{2}\right)+d r^{2}+C(r) d \theta^{2}
$$

The corresponding Eisntein's equations are given in the Appendix. In the weak gravity approximation, $B(r)=1+\beta(r), C(r)=r^{2}[1+\gamma(r)]$, and the linearized equations for $\beta$ and $\gamma$ can be solved in the same way as for monopoles. The resulting metric is

$$
d s^{2}=(1+2 \Phi)\left(-d t^{2}+d z^{2}\right)+d r^{2}+(1+k \Phi) r^{2} d \theta^{2}
$$

Once again, $\Phi(r)$ is the Newtonian potential (2.5) and $k \sim 1$ is a numerical coefficient. For $n=2$, we have $a=2 / 6^{1 / 4}=1.28, K=15.1$, and $k=1.7$.

For a gauge vacuumless string, the energy-momentum tensor for the solution (1.4) is dominated by gradient terms,

$$
T_{0}^{0}=T_{z}^{z}=T_{\theta}^{\theta}=-T_{r}^{r}=\frac{f^{\prime 2}}{2}=\frac{a^{2}}{2 r^{2}} .
$$


The metric can still be expressed in the form (2.11), and the linearized Einstein's equations take the form

$$
\begin{gathered}
\beta^{\prime \prime}+\frac{1}{r} \beta^{\prime}=0, \\
\beta^{\prime \prime}-\frac{1}{r} \beta^{\prime}=8 \pi G \frac{a^{2}}{r^{2}}, \\
\gamma^{\prime \prime}+\frac{2}{r} \gamma^{\prime}+2 \beta^{\prime \prime}=-16 \pi G \frac{a^{2}}{r^{2}} .
\end{gathered}
$$

The solution is easily found, and the resulting metric has the form

$$
d s^{2}=(1+2 \Phi)\left(-d t^{2}+d z^{2}\right)+d r^{2}+(1-4 \Phi) r^{2} d \theta^{2}
$$

Here,

$$
\Phi=2 \pi G a^{2} \ln (r / \delta)
$$

and $a$ is given by Eq. (1.5).

\section{NUMERICAL SOLUTIONS}

To go beyond the linear approximation, we found the gravitational field of vacuumless defects by numerically solving the combined Einstein's and matter field equations.

For a global monopole, we used the metric ansatz (2.6) with the boundary conditions

$$
\begin{gathered}
A(0)=B(0)=1, \\
f(0)=0, \quad f^{\prime}(R)=0 .
\end{gathered}
$$

The parameters of the scalar field potential (1.2) were chosen as $n=2, \lambda=1$, and $M=$

$10^{-2} m_{p}$. The resulting solutions for $f(r), A(r)$, and $B(r)$ are shown in Figs. 1 and 2. The solution for the scalar field $f(r)$ is not much different from the flat space solution with the 
same values of the parameters. Outside the monopole core, the metric coefficient $A(r)$ is very accurately given by

$$
A^{-1}(r)=1-\kappa \lambda^{1 / 2} G M^{3} r
$$

where $\kappa=25.6$. Note that it is in a good agreement with the linearized theory which gives $\kappa=k K=17 \pi / 2$. From Eq. (3.3) we see that $A(r)$ diverges at $r=r_{0}=\left(\kappa \lambda^{1 / 2} G M^{3}\right)^{-1}$. $B(r)$ vanishes at the same point, while the product $A B$ remains finite. The singularity at $r=r_{0}$ is only a coordinate singularity. It is of the same type as the singularity at the horizon, $r=H^{-1}$, in the static de Sitter metric,

$$
d s^{2}=-\left(1-H^{2} r^{2}\right) d t^{2}+\left(1-H^{2} r^{2}\right)^{-1} d r^{2}+r^{2} d \Omega^{2} .
$$

An observer at the monopole center will see test particles accelerating away and "fading away" as they approach the horizon.

We note that $f\left(r_{0}\right) / m_{p}=0.18 \sim 1$. This indicates that the horizons form when the field $f$ becomes comparable to $m_{p}$ in the space between monopoles, that is, when the typical separation between monopoles is $R \sim r_{0}$. It was argued in Ref. [5] that the monopoles start dominating the universe at about the same time. We have verified that the value of $f\left(r_{0}\right) / m_{p}$ is roughly independent of $M$.

For a gauge monopole, the gravitational field at $r \ll R$ is determined mainly by the energy concentration near the core, while the potential energy term is negligible. In flat spacetime we have found [5] that the potential $V(f)$ has a negligible effect on the scalar field as well, which corresponds to the Prasad-Sommerfield (PS) limit. The role of the potential is only to determine the constant $a$ appearing in the PS solution (1.6). We expect the situation in curved spacetime to be similar. As we discussed in [5], when $R$ is increased, $a$ grows, the monopole mass grows, and the size of its core decreases, so that the monopole core becomes a black hole at some critical value $a=a_{c}$. Gravitating monopoles in the PS limit have been studied numerically by Ortiz [6] and by Breitenlohner, Forgács and Maison [7]. They found $a_{c} \approx 0.4 m_{p}$. We obtained numerical solutions for vacuumless gauge monopoles and found essentially identical results. We shall not reproduce them here. 
For a global string, we used the ansatz 2.11) with the boundary conditions

$$
\begin{array}{r}
B(0)=1, \quad C(0)=0, \\
B^{\prime}(0)=C^{\prime}(0)=0,
\end{array}
$$

and Eq. (3.2).

The numerically calculated functions $B(r)$ and $C(r) / r^{2}$ are plotted in Fig. 3. As for a global monopole, $B(r)$ vanishes at a finite distance from the core, $r=r_{0}$, and $C(r)$ diverges on the same surface. However, in the case of string this appears to be a true spacetime singularity, since the invariant $R_{\mu \nu \sigma \tau} R^{\mu \nu \sigma \tau}$ diverges at $r \rightarrow r_{0}$. The scalar field $f(r)$, shown in Fig. 4 , appears to be well-behaved at $r \rightarrow r_{0}$. We find that $f\left(r_{0}\right)=0.16 m_{p}$ and is essentially independent of $M$ (for $M \ll m_{p}$ ). Hence, singularities do not form until $f$ becomes comparable to $m_{p}$ in the space between the strings.

The singularity of a global vacuumless string spacetime is not so surprising if we recall that static spacetimes of "ordinary" global strings exhibit similar singularities [8]. Gregory [9] has recently argued that the singularity can be removed if the space is allowed to expand along the string axis in a de Sitter-like fashion. The same argument may apply to vacuumless strings.

We finally consider a vacuumless gauge string. The functions $B(r)$ and $C(r) / r^{2}$ for such a string are shown in Fig. 5. At small values of $r$ they agree well with the linearized solution (2.17) describing an attractive gravitational potential. The character of the metric changes when the potential $V(f)$ becomes comparable to the gradient terms in $T_{\mu \nu}$. At large $r$ the potential dominates and the gravitational field of the string is repulsive.

As the cutoff radius $R$ is increased, $f(R)$ grows. When $f(R)$ approaches the critical value $f_{c} \approx 0.66 m_{p}$ (roughly independent of $M$ for $M \ll m_{p}$ ), B(r) tends to zero, while $C(r)$ begins to diverge. The curvature invariant $R_{\mu \nu \sigma \tau} R^{\mu \nu \sigma \tau}$ also diverges, indicating a true spacetime singularity. The solutions for $f(r)$ and $\alpha(r)$ are shown in Fig. 6. (The gauge field $\alpha(r)$ is defined in the Appendix.)

To elucidate the nature of this singularity, we attempted to approximate the gauge 
string metric at large $r$ analytically. The flat-space solution (1.4) suggests that $f(r)$ is a slowly-varying function, so one can expect that the potential $V(f)$ will be nearly constant for $r \sim R$. Indeed, numerically we find that $V$ changes very little in this range. An approximate representation of the string metric can then be found by solving Einstein's equation with the cylindrically-symmetric ansatz (2.11) and with a constant vacuum energy source, $V=V_{0}$. The solution is

$$
d s^{2}=\cos ^{4 / 3}(Q r)\left(-d t^{2}+d z^{2}\right)+d r^{2}+Q^{-2} \tan ^{2}(Q r) \cos ^{4 / 3}(Q r) d \theta^{2},
$$

where $Q=\left(32 \pi V_{0} / 3 m_{p}^{2}\right)^{1 / 2}$. It has a geometric singularity at $r=\pi / 2 Q$. The qualitative behavior of this metric near the singularity is similar to that of our numerical solution.

\section{CONCLUSIONS}

We have studied the gravitational fields produced by vacuumless strings and monopoles. For a global monopole, the field is strongly repulsive and the spacetime has an event horizon similar to that in de Sitter space. Only the interior of the horizon is describable by a static metric.

The metric of a gauge vacuumless monopole is very similar to that of ordinary monopole in the Prasad-Sommerfield limit. When the Higgs field outside the monopole reaches a critical value $f_{c} \sim m_{p}$, the spacetime develops an event horizon and the monopole core becomes a black hole.

For a global string, the gravitational field is strongly repulsive and the spacetime becomes singular at a finite distance from the string core.

The gravitational field of a gauge string is attractive at small distances and repulsive at large distances. There is also a spacetime singularity at a finite distance from the core.

The singular nature of the string spacetimes is a cause for some concern. As we argued in [5], vacuumless strings can naturally arise at a phase transition in the early universe. If the evolution of such strings leads to naked singularities, we would have a contradiction with 
the cosmic censorship hypothesis [10] which forbids the formation of naked singularities from regular initial conditions. Once naked singularities are formed, the subsequent evolution of the universe can no longer be determined. No region of space would be immune to this problem, since the string network permeates the entire universe. Hence, the distance from a typical observer to a singularity would be comparable to the average distance between the strings.

A possible way out of this difficulty may be to lift the requirement that the vacuumless string metric should be static. Static spacetimes of ordinary global strings also exhibit singularities [8], and Gregory [9] has argued that these singularities can be removed if the space is allowed to expand in a de Sitter-like fashion along the string axis. (The situation would then be similar to the nonsingular spacetime of a domain wall [11,12] which is expanding in the plane of the wall.) This issue requires further study.

\section{ACKNOWLEDGMENTS}

This work was supported in part by the National Science Foundation.

\section{APPENDIX: FIELD EQUATIONS}

For strings, with the Lagrangian (1.1) and the metric (2.11) Einstein's equations are

$$
\begin{gathered}
\frac{1}{2} \frac{B^{\prime \prime}}{B}+\frac{1}{4} \frac{B^{\prime}}{B} \frac{C^{\prime}}{C}=8 \pi G\left(T_{t}^{t}-\frac{1}{2} T\right) \\
\frac{B^{\prime \prime}}{B}+\frac{1}{2} \frac{C^{\prime \prime}}{C}-\frac{1}{2}\left(\frac{B^{\prime}}{B}\right)^{2}-\frac{1}{4}\left(\frac{C^{\prime}}{C}\right)^{2}=8 \pi G\left(T_{r}^{r}-\frac{1}{2} T\right), \\
\frac{1}{2} \frac{C^{\prime \prime}}{C}+\frac{1}{2} \frac{B^{\prime}}{B} \frac{C^{\prime}}{C}-\frac{1}{4}\left(\frac{C^{\prime}}{C}\right)^{2}=8 \pi G\left(T_{\theta}^{\theta}-\frac{1}{2} T\right),
\end{gathered}
$$

where

$$
T_{t}^{t}=T_{z}^{z}=\frac{f^{\prime 2}}{2}+\frac{f^{2}(1-\alpha)^{2}}{2 C}+\frac{\alpha^{\prime 2}}{2 e^{2} C}+V(f)
$$




$$
\begin{aligned}
& T_{r}^{r}=-\frac{f^{\prime 2}}{2}+\frac{f^{2}(1-\alpha)^{2}}{2 C}-\frac{\alpha^{\prime 2}}{2 e^{2} C}+V(f), \\
& T_{\theta}^{\theta}=\frac{f^{\prime 2}}{2}-\frac{f^{2}(1-\alpha)^{2}}{2 C}-\frac{\alpha^{\prime 2}}{2 e^{2} C}+V(f),
\end{aligned}
$$

and $T=T_{\mu}^{\mu}$. With the string ansatz for the gauge field, $A_{\theta}(r)=-\alpha(r) / e r$, the scalar and gauge field equations are

$$
\begin{gathered}
f^{\prime \prime}+\left(\frac{B^{\prime}}{B}+\frac{C^{\prime}}{2 C}\right) f^{\prime}-\frac{1}{C} f(1-\alpha)^{2}-\frac{\partial V(f)}{\partial f}=0, \\
\alpha^{\prime \prime}+\left(\frac{B^{\prime}}{B}-\frac{C^{\prime}}{2 C}\right) \alpha^{\prime}+e^{2} f^{2}(1-\alpha)=0 .
\end{gathered}
$$

The equations with $\alpha=0$ are for the global string. We numerically solve the above equations with the boundary conditions (3.2), (3.5), $\alpha(0)=0$, and $\alpha(R)=1$.

For monopoles, with the metric (2.6) and the gauge field $A_{i}^{a}=-[1-\omega(r)] \epsilon^{a i j} x^{j} / e r^{2}$ Einstein's equations are

$$
\begin{gathered}
\frac{A^{\prime}}{A^{2} r}-\frac{1}{A r^{2}}+\frac{1}{r^{2}}=8 \pi G\left[\frac{f^{\prime 2}}{2 A}+\frac{f^{2} \omega^{2}}{r^{2}}+\frac{1}{e^{2} r^{2}}\left[\frac{\omega^{\prime 2}}{A}+\frac{\left(1-\omega^{2}\right)^{2}}{2 r^{2}}\right]+V(f)\right], \\
\frac{(A B)^{\prime}}{A B}=16 \pi G r\left(\frac{f^{\prime 2}}{2}+\frac{\omega^{\prime 2}}{e^{2} r^{2}}\right),
\end{gathered}
$$

and the field equations are

$$
\begin{gathered}
\frac{1}{A} f^{\prime \prime}+\frac{1}{A}\left(\frac{B^{\prime}}{2 B}-\frac{A^{\prime}}{2 A}+\frac{2}{r^{2}}\right) f^{\prime}-\frac{2}{r^{2}} \omega^{2} f-\frac{\partial V(f)}{\partial f}=0 \\
\frac{1}{A} \omega^{\prime \prime}+\frac{1}{2 A}\left(\frac{B^{\prime}}{B}-\frac{A^{\prime}}{A}\right) \omega^{\prime}+\frac{\omega\left(1-\omega^{2}\right)}{r^{2}}-e^{2} f^{2} \omega=0 .
\end{gathered}
$$

The equations with $\omega=0$ are for the global monopole. We apply boundary conditions (3.1), (3.2), $\omega(0)=1$, and $\omega(R)=0$. 


\section{REFERENCES}

[1] T.W.B. Kibble, J. Phys. A 9, 1387 (1976).

[2] For a review of topological defects, see, e.g., A. Vilenkin and E.P.S. Shellard, Cosmic Strings and Other Topological Defects (Cambridge Univ. Press, Cambridge, 1994).

[3] I. Affleck, M. Dine, and N. Seiberg, Nucl. Phys. B 241, 493 (1984); P. Binetruy, M. Gaillard, and Y.Y. Wu, Phys. Lett. B 412, 288 (1997).

[4] P.J.E. Peebles and B. Ratra, Ap. J. Lett. 325, L 17 (1988); Phys. Rev. D 37, 3406 (1988); R.R. Caldwell, R. Dave, and P.J. Steinhardt, Phys. Rev. Lett. 80, 1582 (1998);

I. Zlatev, L. Wang, and P.J. Steinhardt, astro-ph/9807002.

[5] I. Cho and A. Vilenkin, hep-th/9808090.

[6] M. Ortiz, Phys. Rev. D 45, R2586 (1992).

[7] P. Breitenlohner, P. Forgács, and D. Maison, Nucl. Phys. B 383, 357 (1992).

[8] A. Cohen and D. Kaplan, Phys. Lett. B 215, 67 (1988); R. Gregory, ibid. 215, 663 (1988); G. Gibbons, M. Ortiz and F. Ruiz, Phys. Rev. D 39, 1546 (1989).

[9] R. Gregory, Phys. Rev. D 54, 4955 (1996).

[10] R. Penrose, Rivista del Nuovo Cimento 1, 252 (1969).

[11] A. Vilenkin, Phys. Lett. B 133, 177 (1983).

[12] J. Ipser and P. Sikivie, Phys. Rev. D 30, 712 (1984). 


\section{FIGURES}

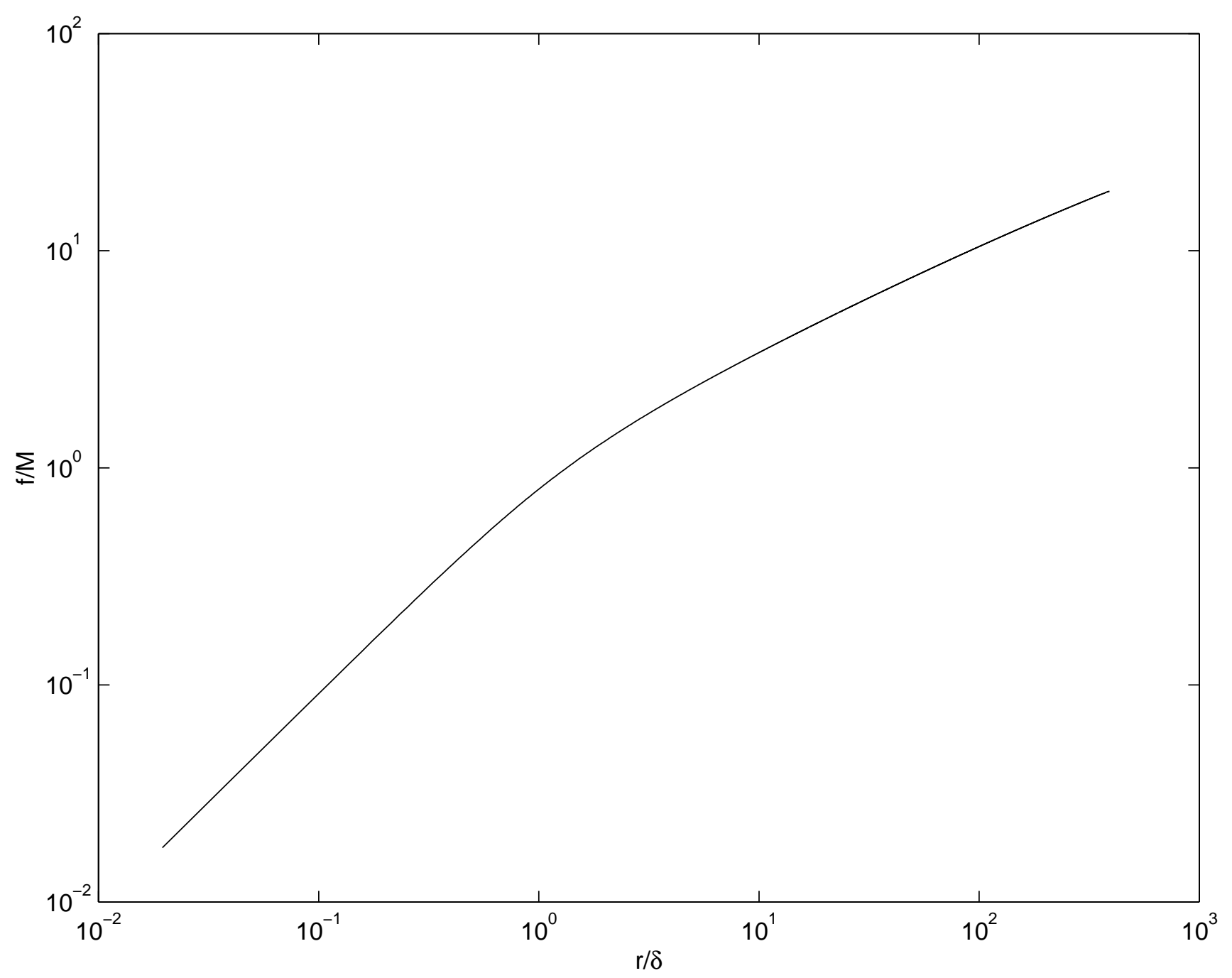

FIG. 1. Scalar field $f(r)$ of a vacuumless global monopole with $M=0.01 m_{p}$. 


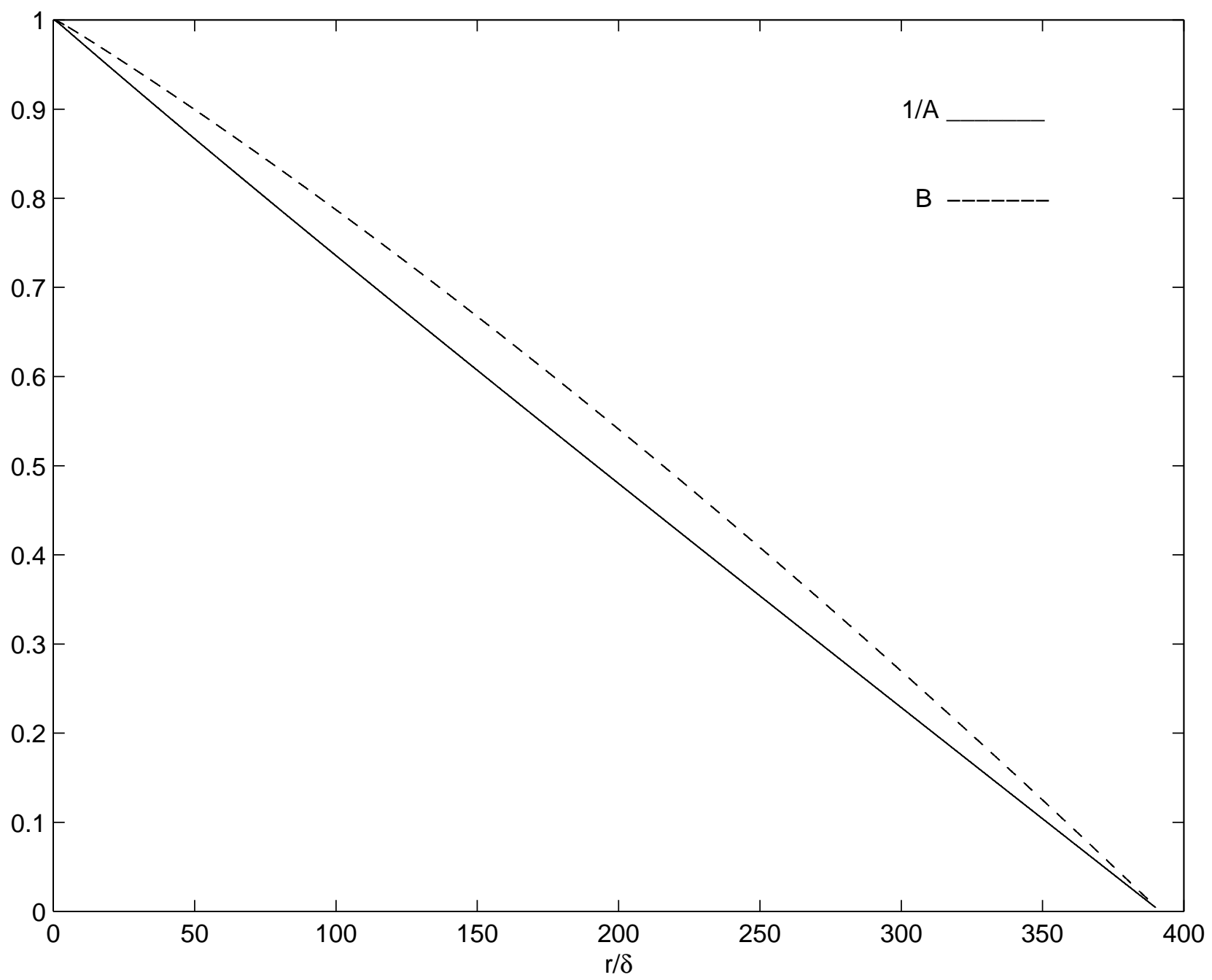

FIG. 2. Metric coefficients $B(r)$ and $1 / A(r)$ of a vacuumless global monopole with $M=0.01 m_{p}$. 


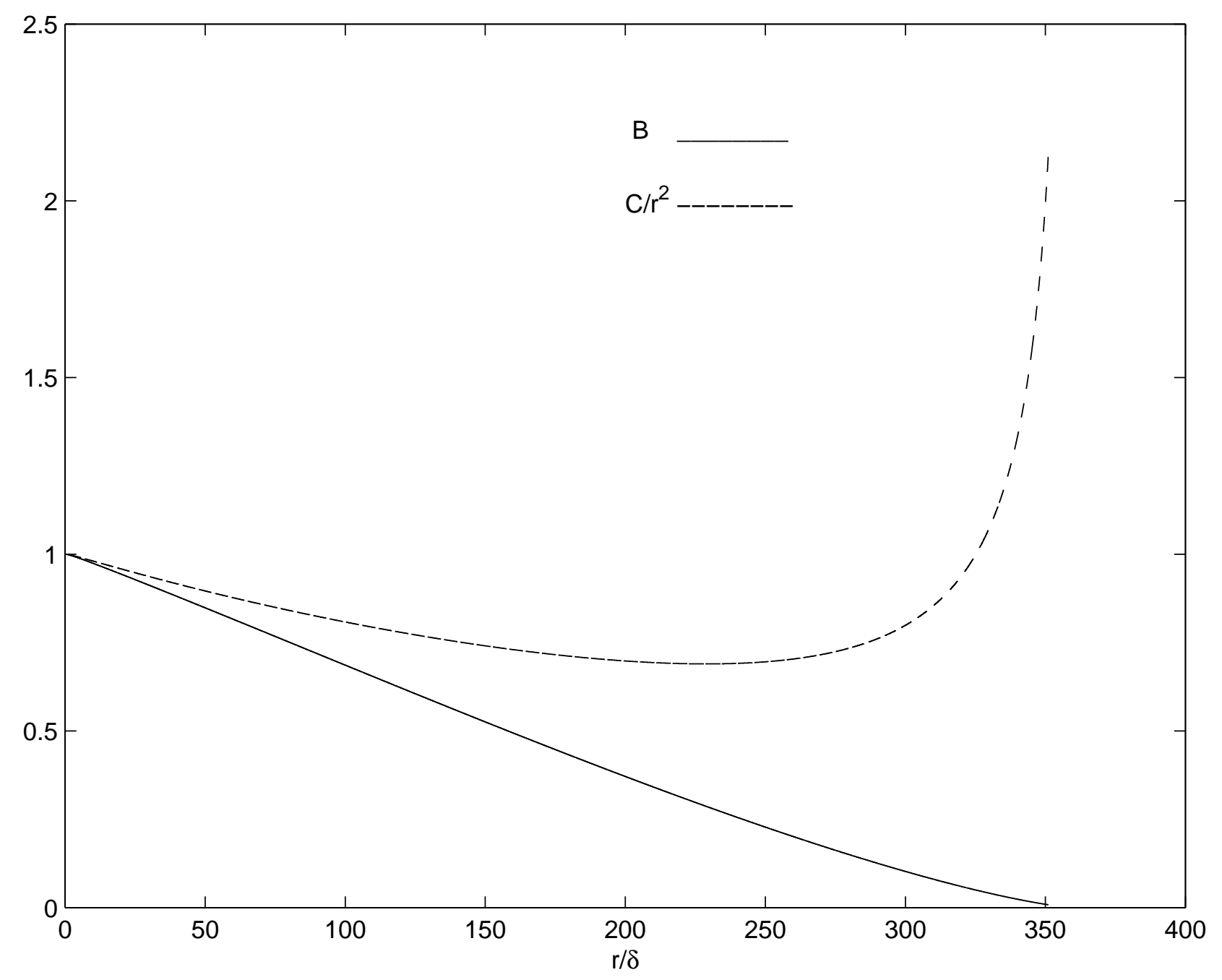

FIG. 3. Metric coefficients $B(r)$ and $C(r) / r^{2}$ of a vacuumless global string with $M=0.01 m_{p}$. 


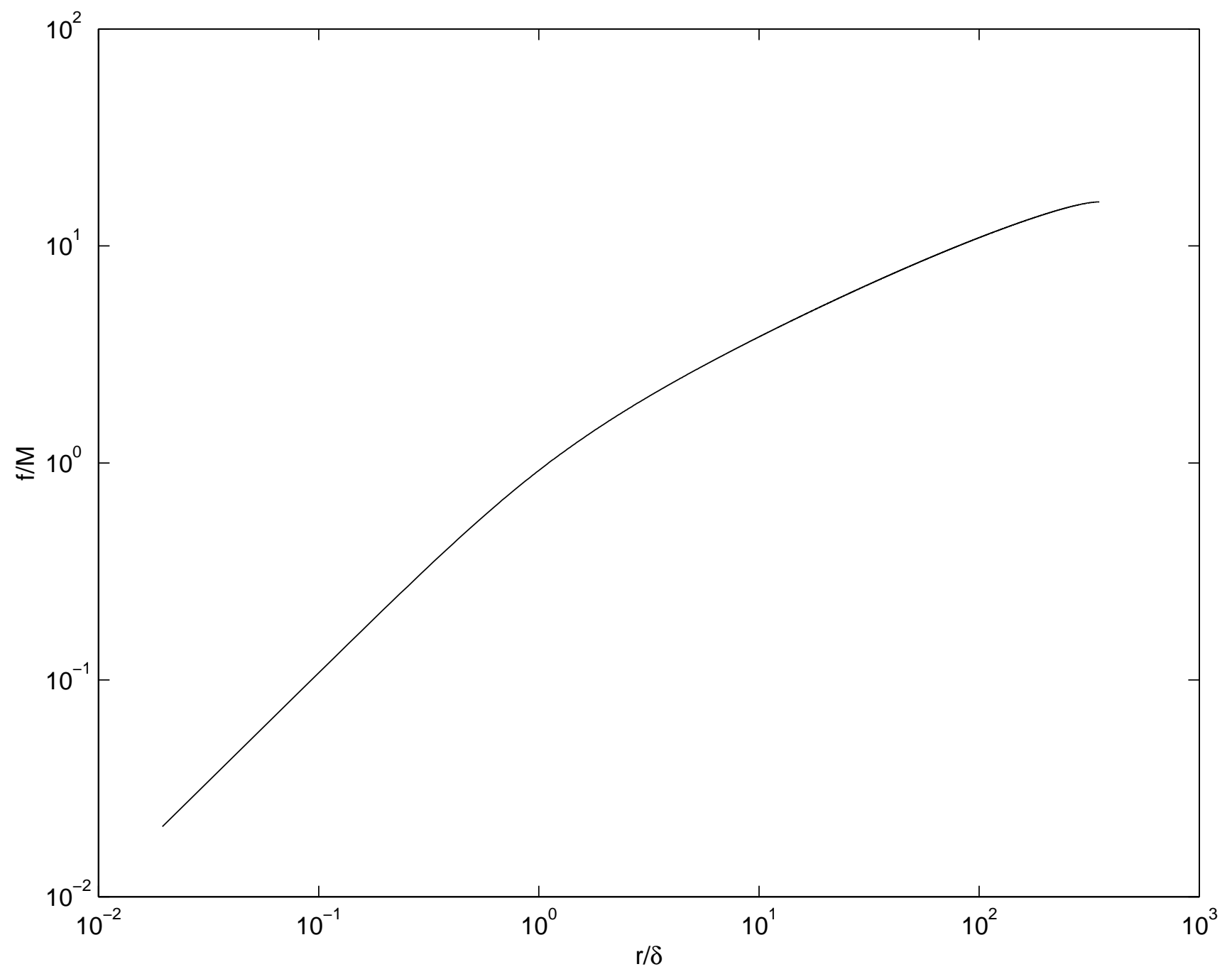

FIG. 4. Scalar field $f(r)$ of a vacuumless global string with $M=0.01 m_{p}$. 


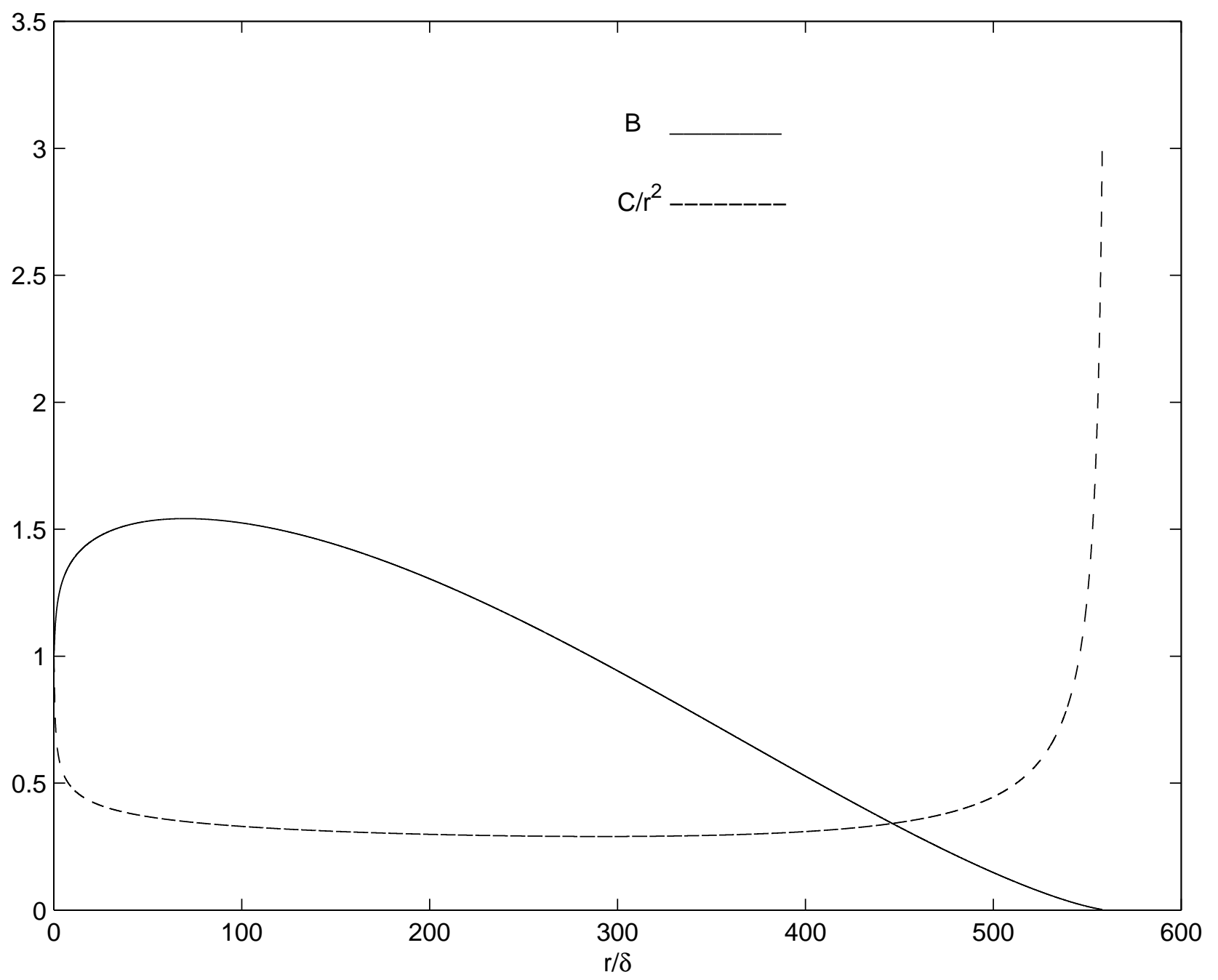

FIG. 5. Metric coefficients $B(r)$ and $C(r) / r^{2}$ of a vacuumless gauge string with $M=0.02 m_{p}$ and $\lambda / e^{2}=1$. 


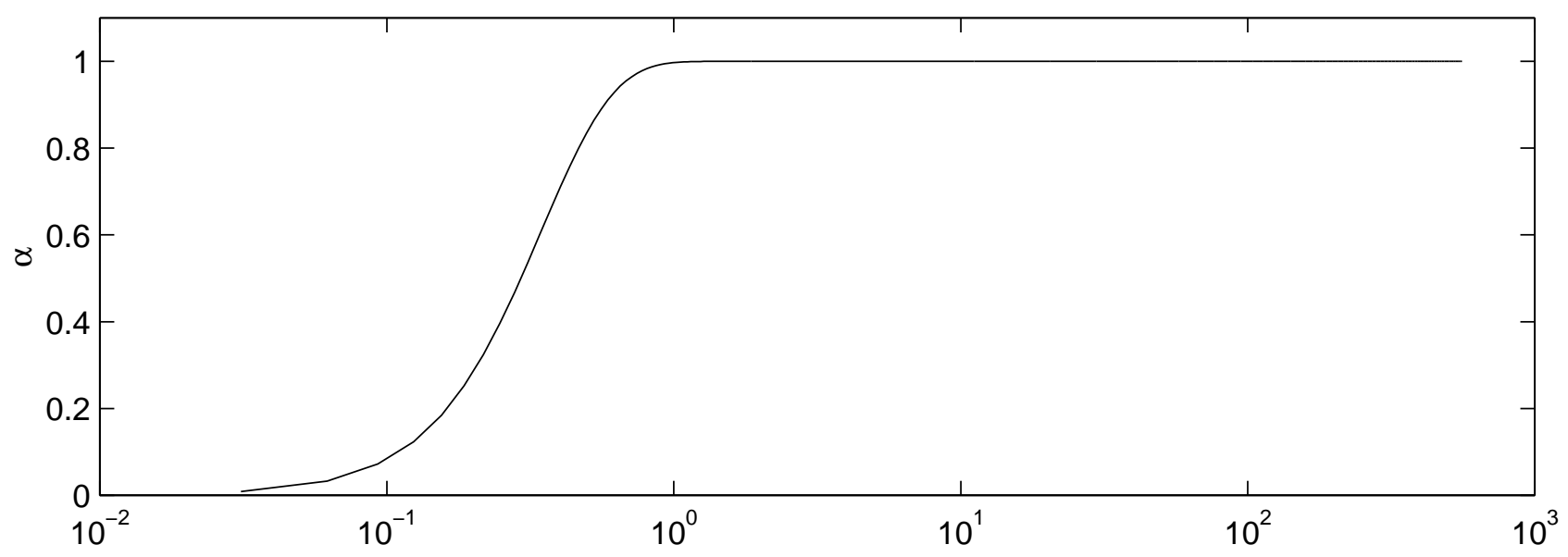

(a)

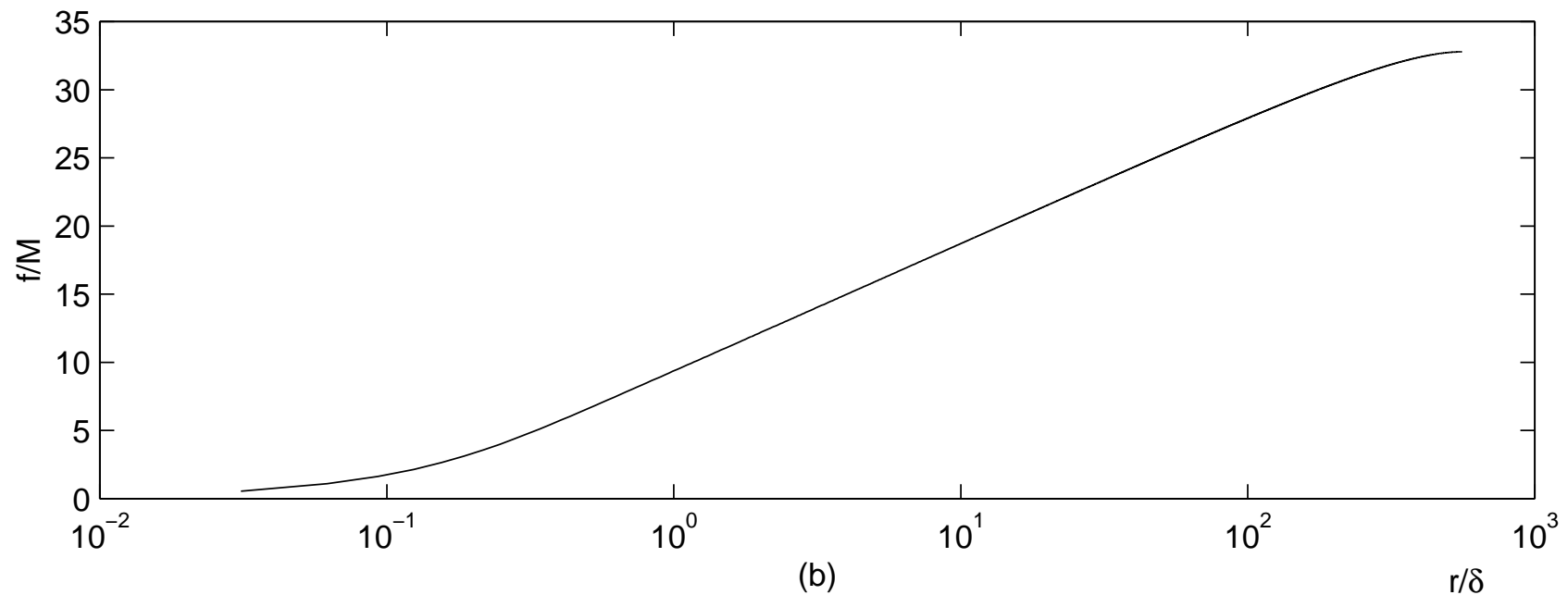

FIG. 6. (a) Gauge field $\alpha(r)$ and (b) scalar field $f(r)$ of a gauge vacuumless string with $M=0.02 m_{p}$ and $\lambda / e^{2}=1$. 